\title{
Brief
}

\section{High-dose aprotinin effectively reduces blood loss during on-pump coronary artery bypass grafting with bivalirudin anticoagulation}

\author{
Andreas Koster, MD, ${ }^{a}$ Semih Buz, MD, ${ }^{\mathrm{b}}$ Thomas Krabatsch, MD, ${ }^{\mathrm{b}}$ Frank Dehmel, ${ }^{\mathrm{c}}$ Hermann Kuppe, MD, ${ }^{\mathrm{a}}$ \\ Roland Hetzer, MD, ${ }^{\mathrm{b}}$ Solomon Aronson, MD, ${ }^{\mathrm{d}}$ and Cornelius M. Dyke, MD, ${ }^{\mathrm{e}}$ Berlin, Germany, Durham and \\ Gastonia, NC
}

See related articles on pages 487, 495, and 573.

From the Departments of Anesthesia, ${ }^{\text {a }}$ Cardiothoracic and Vascular Surgery , ${ }^{b}$ and Perfusion, ${ }^{\mathrm{c}}$ Deutsches Herzzentrum, Berlin, Germany; Department of Anesthesia, Duke University Medical Center, ${ }^{d}$ Durham, NC; and Gaston Memorial Hospital, ${ }^{\mathrm{e}}$ Gastonia, NC.

Work attribution: Department of Anesthesia, Deutsches Herzzentrum Berlin, Germany.

The study was supported by The Medicines Company, Parsippany, New Jersey. Drs Koster, Dyke, and Aronson report consulting fees from The Medicines Company. Dr Dyke reports ownership of equity in The Medicines Company.

Received for publication July 10, 2007; revisions received July 31, 2007; accepted for publication Sept 7, 2007.

Address for reprints: Andreas Koster, MD, Deutsches Herzzentum Berlin, Augustenburger Platz 1, D-13353 Berlin (E-mail: koster@dhzb.de).

J Thorac Cardiovasc Surg 2008;135:685-7

$0022-5223 / \$ 34.00$

Copyright (C) 2008 by The American Association for Thoracic Surgery

doi:10.1016/j.jtcvs.2007.09.018

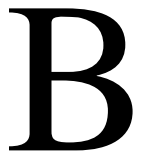

ivalirudin is a short-acting direct thrombin inhibitor that is increasingly used for anticoagulation during cardiac surgery in patients with heparin-induced thrombocytopenia (HIT). ${ }^{1}$ During cardiopulmonary bypass (CPB) with heparin anticoagulation, aprotinin reduces hemostatic activation, inflammatory response, perioperative blood loss, and transfusion requirements. The current investigation was performed to assess the effects of aprotinin when bivalirudin is used for anticoagulation during CPB.

\section{Materials and Methods}

After approval by the German Ministry for Drug Safety and the local ethics committee and informed consent was obtained, 14 patients scheduled for first-time elective coronary artery bypass grafting $(\mathrm{CABG})$ were enrolled in this prospective single-center investigation. Patients were randomized to 2 groups with 7 patients each: 1 group with bivalirudin anticoagulation only and 1 group with bivalirudin and aprotinin. All patients had normal renal function, a left ventricular ejection fraction of more than $30 \%$, and antiplatelet therapy discontinued 5 days before surgery. Bivalirudin dosing and CPB management were performed as described before using closed CPB systems without cardiotomy suction. ${ }^{2}$ Aprotinin was given according to a highdose protocol with $2 \times 10^{6}$ kallikrein inhibiting units (KIU) as patient bolus, $2 \times 10^{6} \mathrm{KIU}$ in the CPB priming solution, and continuous infusion of $0.5 \times 10^{6} \mathrm{KIU}$ during CPB. Packed red blood cell concentrates were transfused when the hemoglobin level was less than $8 \mathrm{~g} / \mathrm{dL}$. The transfusion of fresh-frozen plasma and platelets was based on the physicians' decision. Samples for measurement of bivalirudin concentrations were obtained after the bolus was given and at intervals of 15 minutes during $\mathrm{CPB}$, at intervals of 5 minutes until 1 hour after CPB, and at intervals of 15 minutes during the following hour. Bivalirudin concentrations were analyzed with high-performance liquid chromatography. Samples for assessment of markers of hemostatic activation (fibrinopeptide A and prothrombin fragment 1+2) and inflammation (interleukin 6 and myeloperoxidase) were obtained 5 minutes after the bivalirudin bolus was given (before initiation of $\mathrm{CPB}$ ) and after termination of the bivalirudin infusion, shortly before termination of CPB. Samples were analyzed with standard enzyme-linked immune assays. Statistical analysis was performed using the Student $t$, Wilcoxon rank-sum, and paired Wilcoxon signed-rank tests.

\section{Results}

There were no differences regarding patients' demographic data, duration of CPB, or surgery (Table 1). The clinical course of all patients was uneventful. In control patients treated only with bivalirudin, there was a moderate increase in markers of hemostatic activation and inflammatory response during CPB. The administration of high-dose aprotinin did not influence bivalirudin pharmacokinetics, hemostatic activation, and inflammatory response, but there was a significant reduction of perioperative blood loss and a marked trend toward lower transfusion requirements (Table 1; Figure 1). 


\section{Abbreviations and Acronyms}

$\mathrm{CABG}=$ coronary artery bypass grafting

$\mathrm{CPB}=$ cardiopulmonary bypass

HIT = heparin-induced thrombocytopenia

KIU $=$ kallikrein inhibiting unit

\section{Discussion}

The elimination of bivalirudin is mainly achieved by proteolytic cleavage. Because aprotinin is a proteinase inhibitor, concerns may be raised that high concentrations of this agent may prolong bivalirudin half-life and thereby increase the risk of hemorrhage. In a previous pilot investigation using a different protocol for bivalirudin and a half-dose aprotinin regimen, no impact of aprotinin on bivalirudin pharmacokinetics, blood loss, and transfusion requirements was noted. ${ }^{3}$ Our current data show that a high-dose aprotinin protocol does not affect the pharmacology of bivalirudin. Moreover, the administration of aprotinin does not attenuate the moderate hemostatic activation and inflammatory re- sponse observed during bivalirudin anticoagulation of CPB. However, even in this small number of patients, the administration of aprotinin according to the high-dose protocol resulted in a highly significant decrease in perioperative blood loss and a marked trend toward a reduction of transfusions.

Aprotinin is one of the most effective drugs to reduce perioperative blood loss and transfusion requirements in patients undergoing CPB procedures with heparin anticoagulation. Recent studies associated the use of aprotinin with increased myocardial infarction, renal failure, and 5-year mortality when given during $\mathrm{CPB}$ with heparin anticoagulation and protamine reversal in patients undergoing elective CABG. ${ }^{4,5}$ These results may promote the more cautious use of aprotinin, particularly in low-risk patients undergoing CABG. However, although the current investigation was not powered to assess patient outcomes in this regard, the observed reduction of blood loss and transfusions in our patients undergoing on-pump CABG with bivalirudin anticoagulation remains impressive. The effects of drugs influencing the hemostatic system observed in patients without HIT cannot automatically be translated to patients with HIT. Nevertheless, in view of the current data, we believe that in the high-risk population of patients with HIT,

TABLE 1. Clinical and laboratory data

\begin{tabular}{|c|c|c|c|}
\hline & $\begin{array}{l}\text { Bivalirudin } \\
(n=7)\end{array}$ & $\begin{array}{l}\text { Bivalirudin }+ \text { aprotinin } \\
\qquad(\mathrm{n}=7)\end{array}$ & $\boldsymbol{P}$ \\
\hline \multicolumn{4}{|l|}{ Baseline characteristics } \\
\hline Age & $63.0 \pm 7.0$ & $61.7 \pm 9.1$ & .70 \\
\hline Gender, male \% & 85.7 & 85.7 & 1.0 \\
\hline Weight $(\mathrm{kg})$ & $88.7 \pm 15.0$ & $89.0 \pm 14.9$ & .85 \\
\hline \multicolumn{4}{|l|}{ CPB + surgery } \\
\hline Time on CPB (min) & $86.4 \pm 21.4$ & $93.0 \pm 28.2$ & .57 \\
\hline Duration of surgery (min) & $213.9 \pm 38.4$ & $214.7 \pm 54.8$ & .85 \\
\hline \multicolumn{4}{|l|}{ Blood loss + transfusions } \\
\hline Mean blood loss $2 \mathrm{~h}$ postsurgery $(\mathrm{mL})$ & $454 \pm 304$ & $96 \pm 37$ & .002 \\
\hline Mean blood loss $12 \mathrm{~h}$ postsurgery $(\mathrm{mL})$ & $959 \pm 472$ & $274 \pm 53$ & .002 \\
\hline Any transfusions $(\%)$ & 71.4 & 42.9 & .59 \\
\hline PRBC $(\%)$ & 57.1 & 28.6 & .59 \\
\hline Mean no. of units in patients with PRBC & $3.3 \pm 2.6$ & $2 \pm 0$ & .62 \\
\hline \multicolumn{4}{|l|}{ Inflammation + hemostatic activation } \\
\hline MPO (pM) pre-CPB & $66.8 \pm 136.7$ & $69.4 \pm 153.0$ & .65 \\
\hline MPO (pM) post-CPB & $63.5 \pm 88.4$ & $76.7 \pm 133$ & .95 \\
\hline Pre vs post-CPB $P$ value (within group) & .56 & .237 & \\
\hline IL6 (pg/mL) pre-CPB & $13.0 \pm 4.7$ & $15.9 \pm 11.4$ & .85 \\
\hline IL6 (pg/mL) post-CPB & $72.1 \pm 49.4$ & $83.6 \pm 42.1$ & .57 \\
\hline Pre vs post-CPB $P$ value (within group) & .018 & .028 & \\
\hline FPA (ng/mL) pre-CPB & $20.6 \pm 19.6$ & $14.2 \pm 8.1$ & .85 \\
\hline FPA (ng/mL) post-CPB & $26.0 \pm 16.6$ & $32.6 \pm 19.6$ & .41 \\
\hline Pre vs post-CPB $P$ value (within group) & .091 & .063 & \\
\hline PF $1+2(p M)$ pre-CPB & $476 \pm 264$ & $222 \pm 54$ & .025 \\
\hline PF $1+2(p M)$ post-CPB & $512 \pm 251$ & $418 \pm 211$ & .37 \\
\hline Pre vs post-CPB $P$ value (within group) & .128 & .043 & \\
\hline
\end{tabular}

$C P B$, Cardiopulmonary bypass; $P R B C$, packed red blood cell concentrates; $M P O$, myeloperoxidase; $I L$, interleukin; $F P A$, fibrinopeptide $\mathrm{A}$; $P F 1+2$, prothrombin fragment $1+2$. 


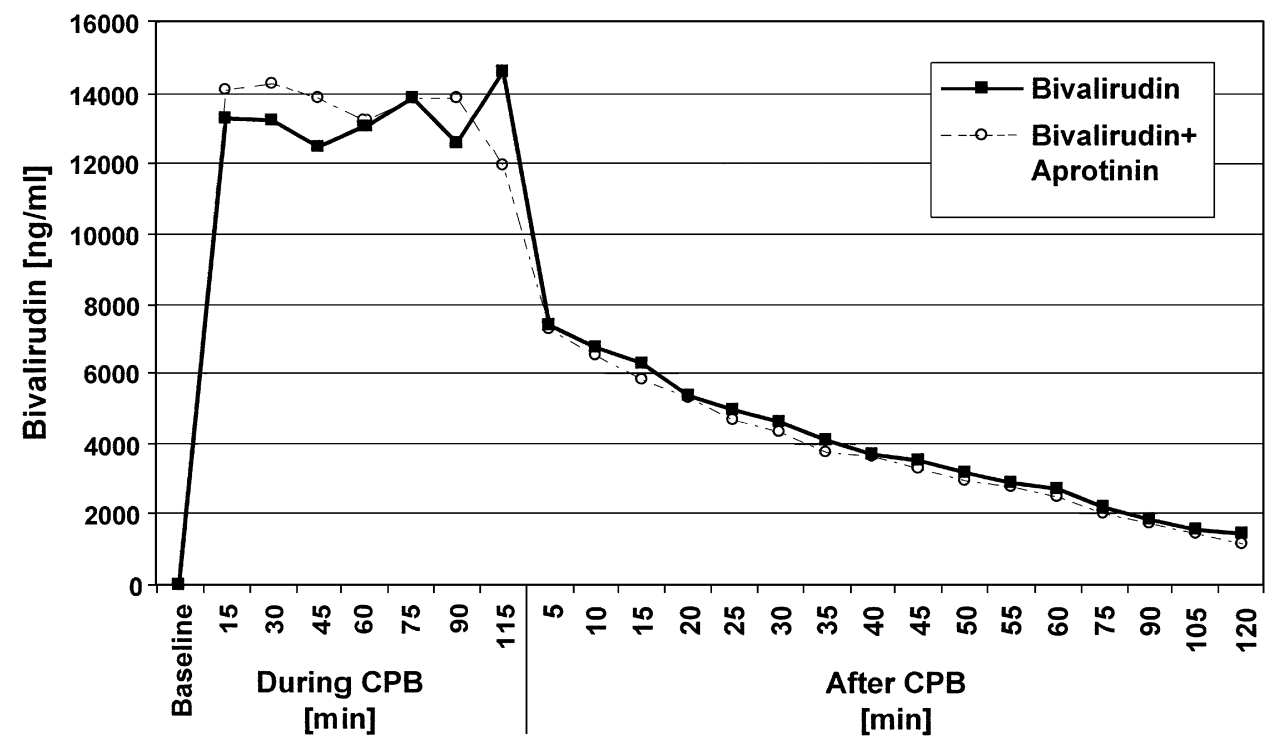

Figure 1. Group 1 = bivalirudin; Group II = bivalirudin + aprotinin.

high-dose aprotinin may be considered as an adjunctive agent during CPB anticoagulation with bivalirudin. This applies particularly to complex procedures with a high risk of increased perioperative bleeding.

\section{References}

1. Koster A, Dyke CM, Aldea G, et al. Bivalirudin during cardiopulmonary bypass in patients with previous or acute heparin-induced thrombocytopenia and heparin antibodies: results of the CHOOSE-ON trial. Ann Thorac Surg. 2007;83:572-7.
2. Koster A, Yeter R, Buz S, et al. Assessment of hemostatic activation during cardiopulmonary bypass for coronary artery bypass grafting with bivalirudin: results of a pilot study. J Thorac Cardiovasc Surg. 2005; 129:1391-4.

3. Koster A, Spiess BD, Chew DP, et al. Effectiveness of bivalirudin as a replacement for heparin during cardiopulmonary bypass in patients undergoing coronary artery bypass grafting. Am J Cardiol. 2004;93:356-9.

4. Mangano DT, Tudor IC, Dietzel C, Multicenter Study of Perioperative Research Group, Ischemia Research and Education Foundation. The risk associated with aprotinin in cardiac surgery. N Engl J Med. 2006;354:353-65.

5. Mangano DT, Miao Y, Vuylsteke A, et al. Mortality associated with aprotinin during 5 years following coronary artery bypass graft surgery. JAMA. 2007;297:471-9. 